\title{
Removal of Polymer for Nano-imprint Lithography using Atomic Hydrogen Generated by the Hot-wire Catalyzer
}

\author{
Akihiko Kono ${ }^{*}$, Takeshi Maruoka ${ }^{*}$, Yu Arai ${ }^{*}$, Yoshihiko Hirai ${ }^{* *}$ and Hideo Horibe ${ }^{*, * * *}$ \\ ${ }^{*}$ Research Laboratory for Integrated Technological Systems, Kanazawa Institute of Technology, 3-1 \\ Yatsukaho, Hakusan, Ishikawa 924-0838, Japan \\ ** Department of Physics and Electronics Engineering, Graduate School of Engineering, \\ Osaka Prefecture University, 1-1 Gakuen-cho, Sakai, Osaka 599-8531, Japan \\ ${ }^{* * *}$ The Institute of Scientific and Industrial Research, Osaka University, 8-1 Mihogaoka, Ibaraki, \\ Osaka 567-0047, Japan \\ a.kono@neptune.kanazawa-it.ac.jp
}

\begin{abstract}
We investigated the removal characteristics of an ultraviolet (UV) curable polymer for UV nano-imprint lithography (NIL) using atomic hydrogen, which was generated by the catalytic decomposition of $\mathrm{H}_{2}$ molecules in $\mathrm{H}_{2} / \mathrm{N}_{2}$ mixed gas $\left(\mathrm{H}_{2}: \mathrm{N}_{2}=10: 90 \mathrm{vol} . \%\right)$ using a tungsten hot-wire catalyzer. The cured polymers were removed by atomic hydrogen without a residual layer. The removal rate of the polymer increased with rising catalyzer temperature. It was assumed that both enhanced reactivity between atomic hydrogen and polymer by the substrate temperature increase due to radiation heat of the catalyzer and increased atomic hydrogen concentration occurred as a result of the rise in catalyzer temperature. When the distance between the catalyzer and the substrate was $100 \mathrm{~mm}$, the catalyzer temperature was $2400^{\circ} \mathrm{C}$, and the initial substrate temperature was room temperature, the removal rate of polymers was $0.17 \mu \mathrm{m} / \mathrm{min}$ independent of exposure dose to the polymer. The removal rate of polymers was comparable to the removal rate of general $i$-line positive-tone novolak resist. The polymer adhering to the micro-asperity portion of the mold was removed without leaving a residual layer by grazing-incidence atomic hydrogen.
\end{abstract}

Keywords: Atomic hydrogen, Hot-wire catalyzer, Nano-imprint lithography,

Polymer removal, Mold cleaning

\section{Introduction}

Recently, nano-imprint lithography (NIL), proposed by Chou et al. [1], has received much attention from many industries because of the potential high throughput production of various nanostructure applications, such as electronic devices with a simple process and low cost. Thermal NIL $[1,2]$ using thermoplastic polymer and ultraviolet (UV) NIL [3, 4] using UV curable polymer have been performed. Figure 1 illustrates the UV-NIL process. First, the substrate is coated with UV curable polymer. Second, a quartz mold with a nano-size pattern is pressed onto the UV curable polymer. Third, UV light is irradiated through the mold to cure the UV curable polymer. Fourth, after the polymer is cured, the mold is removed from the polymer. Finally, the residual polymer layer equivalent to convex portions of the mold is removed by oxygen reactive ion etching, and substrate etching is performed.

One problem with UV-NIL is that polymer adheres to the mold when the mold is removed [5, 6]. This leads to contamination of the mold and abrasion of the patterns transferred from the substrate. The molds are generally prepared by electron beam lithography, and those with high 


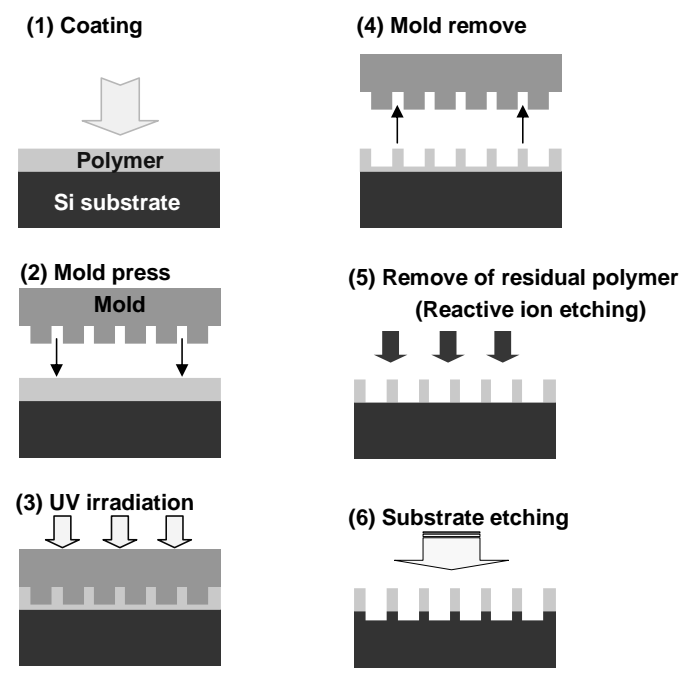

Fig. 1. Schematic diagram of the UV nano-imprint lithography (NIL) process.

aspect ratio patterns are fabricated by the Lithographie Galvanoformung Abformung (LIGA) process using synchrotron radiation. These molds are quite valuable because they are difficult to fabricate. Thus, cleaning technology for removing the adhering polymer to reuse the mold is an elemental technology in UV-NIL. However, the polymer cured by UV irradiation is difficult to remove by conventional methods such as wet processes using chemicals.

In this study, we investigated removing UV curable polymer using UV-NIL by atomic hydrogen generated by catalytic decomposition of $\mathrm{H}_{2}$ molecules using a tungsten hot-wire catalyzer [7-11]. This method is beneficial to the environment because it does not use chemicals such as sulfuric acid hydrogen peroxide mixture (SPM), ammonia hydrogen peroxide mixture (APM), and organic amine solutions. Moreover, it is expected that the nano-sized pattern on the mold will not be damaged by bombardment of high-energy ions because this method is plasma-free.

\section{Experimental}

2-1. Atomic Hydrogen Irradiation Apparatus and Conditions

Figure 2 schematically illustrates the atomic hydrogen irradiation apparatus. A tungsten wire (99.95\% purity, $0.7 \mathrm{~mm}$ in diameter, and $500 \mathrm{~mm}$ in length) was used as a hot-wire catalyzer to generate atomic hydrogen. A 40mm-long, 10mm-diameter catalyzer was coiled into a helix with five turns.

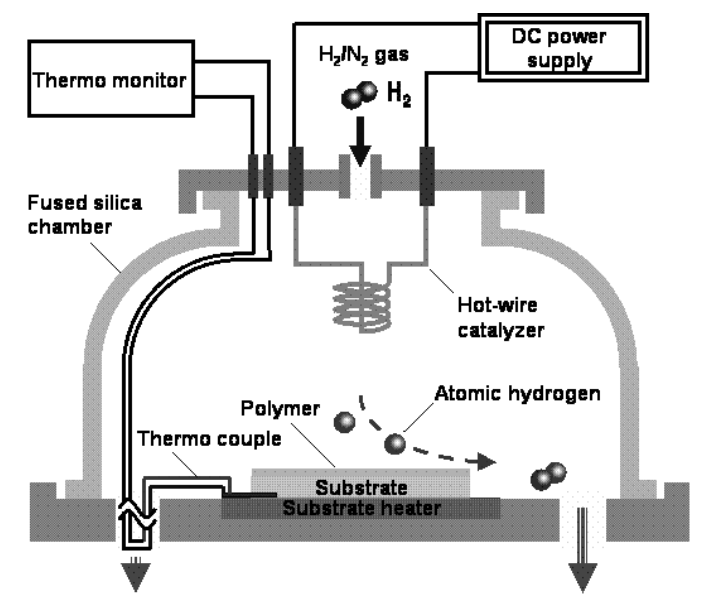

Fig. 2. Schematic illustration of the atomic hydrogen irradiation apparatus.

The polymer-coated Si substrate and the mold with adhering polymer were attached just below the catalyzer. The distance between the catalyzer and the substrate was fixed at $100 \mathrm{~mm}$. The catalyzer was heated at $2000^{\circ} \mathrm{C}$ (current $\left.=20.5 \mathrm{~A}\right), 2100^{\circ} \mathrm{C}$ (22.2A), $2300^{\circ} \mathrm{C}(25.7 \mathrm{~A})$, and $2400^{\circ} \mathrm{C}(27.3 \mathrm{~A})$ using a direct current supply. The catalyzer temperature was measured using a two-wavelength $(0.8$ and $1.05 \mu \mathrm{m})$ infrared radiation thermometer (Impac Electronic ISR12-L0). The working gas, $\mathrm{H}_{2} / \mathrm{N}_{2}$ mixed gas $\left(\mathrm{H}_{2}: \mathrm{N}_{2}=10: 90 \mathrm{vol} . \%\right)$, was introduced from the center nozzle into a fused silica chamber. To avoid explosion, $\mathrm{N}_{2}$ gas was used to dilute the $\mathrm{H}_{2}$ gas concentration. The gas flow rate was fixed at $300 \mathrm{sccm}$ using a mass flow controller. The $\mathrm{H}_{2}$ partial pressure was fixed at $2.13 \mathrm{~Pa}$, and the initial substrate temperature (just below the atomic hydrogen irradiation) was fixed at room temperature (R.T.). The substrate temperature during atomic hydrogen irradiation was monitored using a K-type thermocouple attached to the back of the substrate.

\section{2-2. Preparing Samples for Measuring the Removal Rate}

UV curable polymer PAK-01 manufactured by Toyo Gosei Co. was used. The polymer was spin-coated onto a Si wafer (substrate) using a spin coater (MIKASA MS-A 100) at 4000rpm for 20sec. The polymer was exposed to UV (mercury lamp $i$-line, $365 \mathrm{~nm}$ wavelength, $9 \mathrm{~nm}$ half width, $0.10 \mathrm{~mW} / \mathrm{cm}^{2}$ intensity) in $\mathrm{N}_{2}$ at atmospheric pressure using a mask aligner (MIKASA M-1s). The exposure dose was controlled by changing exposure time. The film thickness of the polymer 
was measured using a stylus surface-profile measurement instrument (ULVAC DekTak 6M). The residual layer of polymer was evaluated by observing the substrate surface using an optical microscope (Nikon ECLIPSE L150) and x-ray photoemission spectroscopy (XPS, SHIMADZU ESCA-3400) measurement of the substrate surface.

\section{2-3. Preparing Samples for Evaluating Mold} Cleaning Performance

UV curable polymer PAK-01 was used. Both the Si mold and the quartz substrate were cleaned with water, acetone (20min), SPM (sulfuric acid:hydrogen peroxide $=1: 1,40 \mathrm{~min}$ ), and UV ozone (5min). The polymer was spin-coated onto a quartz substrate using a spin coater at $1000 \mathrm{rpm}$ for $30 \mathrm{sec}$. The mold was pressed onto the polymer, and the substrate was irradiated with UV light to cure the polymer. The samples (molds with polymer adhering) were prepared by the mold removal. The cleanliness of the mold was evaluated by observing the surface and cross sectional area of the mold using an optical microscope and a scanning electron microscope (SEM, Hitachi High-Technologies TM-1000), and XPS measurement of the mold surface.

\section{Results and discussion}

3-1. Using Atomic Hydrogen to Remove Polymer Coated onto the Si Substrate

Figure 3 plots the catalyzer temperature versus the polymer removal rate. The exposure dose to polymer was fixed at $100 \mathrm{~mJ} / \mathrm{cm}^{2}$ (recommended exposure for PAK-01). Removal rates were calculated by dividing the reduced film thickness by the atomic hydrogen irradiation time. The atomic hydrogen irradiation time was fixed at $30 \mathrm{~min}$. For catalyzer temperatures of 2000 to $2400^{\circ} \mathrm{C}$, the polymer film thickness decreased with atomic hydrogen irradiation, confirming that atomic hydrogen can remove the cured polymer. Based on a report by Izumi et al. [12], who investigated removal characteristics of photo-resists using atomic hydrogen, it is speculated that atomic hydrogen decomposes polymer to $-\mathrm{C}_{\mathrm{x}} \mathrm{H}_{\mathrm{y}}$ and $-\mathrm{OH}$ compounds. The $\mathrm{N}_{2}$ molecules in the working gas do not contribute to the decomposition of polymer. The generation efficiency of the nitrogen atom by a hot-wire catalyzer is much lower than that of the atomic hydrogen. Umemoto et al. found that the atomic hydrogen concentration reaches $10^{14} \mathrm{~cm}^{-3}$ [13] at a catalyzer temperature of $1900^{\circ} \mathrm{C}$ and $\mathrm{H}_{2}$ pressure of

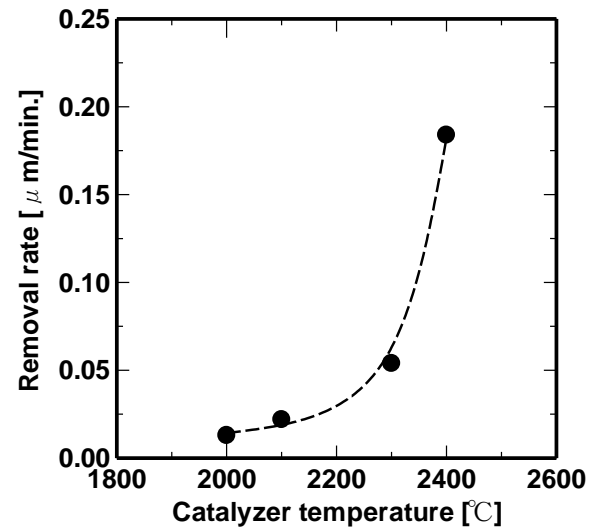

Fig. 3. Relationship between catalyzer temperature and polymer removal rate with an exposure dose of $100 \mathrm{~mJ} / \mathrm{cm}^{2}$.

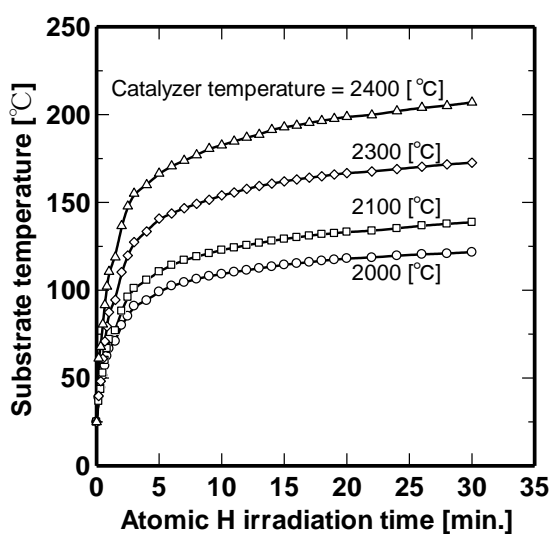

Fig. 4. Substrate temperature change as a function of atomic hydrogen irradiation time. Catalyzer temperatures are $2000,2100,2300$, and $2400^{\circ} \mathrm{C}$.

5.6Pa; however, the nitrogen atom concentration is only $10^{11} \mathrm{~cm}^{-3}$ [14] even at a catalyzer temperature of $2500^{\circ} \mathrm{C}$ and $\mathrm{N}_{2}$ pressure of $100 \mathrm{~Pa}$.

The removal rate rapidly increased with rising catalyzer temperature (Fig. 3). This result is discussed in detail below. Figure 4 presents substrate temperature changes during atomic hydrogen irradiation. For all catalyzer temperatures, substrate temperatures rose with increasing atomic hydrogen irradiation time, and were saturated when the atomic hydrogen irradiation time was longer than 20min. The saturated substrate temperature increased with higher catalyzer temperature because the substrate is heated more by the radiation heat of hot-wire catalyzer as the catalyzer temperature rises. Therefore, the reactivity between atomic hydrogen and polymer is enhanced by the rise of catalyzer temperature, because the reaction 

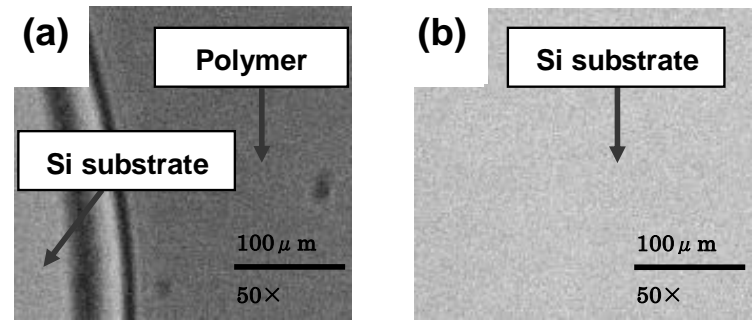

Fig. 5. Optical microscope images $(\times 50)$ of the substrate surface (a) before polymer removal and (b) after polymer removal. The catalyzer temperature is $2400^{\circ} \mathrm{C}$, and the exposure dose to polymer is $100 \mathrm{~mJ} / \mathrm{cm}^{2}$.

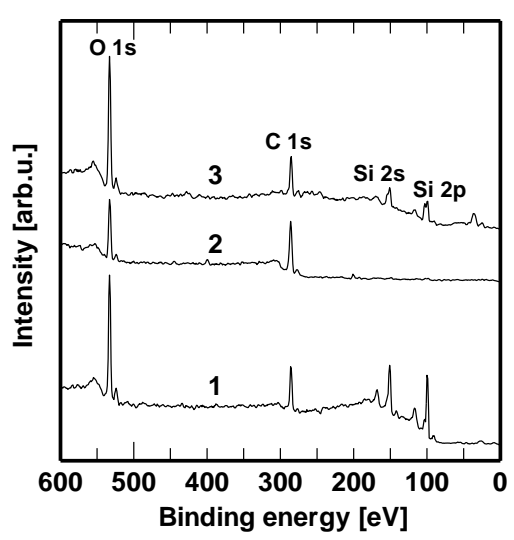

Fig. 6. Wide scan XPS spectra of (1) the Si substrate surface before polymer coating, (2) polymer surface coated onto Si substrate, and (3) Si substrate surface after polymer removal. The catalyzer temperature is $2400^{\circ} \mathrm{C}$, and the exposure dose to polymer is $100 \mathrm{~mJ} / \mathrm{cm}^{2}$.

field temperature rises with catalyzer temperature. Moreover, Umemoto et al. [13] and the authors [10] reported that the natural logarithm of the atomic hydrogen concentration is inversely proportional to the inverse of the absolute catalyzer temperature, and the generation of atomic hydrogen by the hot-wire catalyzer follows the Arrhenius law. The atomic hydrogen concentration increases with rising catalyzer temperature because the atomic hydrogen dissociatively adsorbed onto tungsten dangling bonds of the catalyzer surface easily desorbs at higher catalyzer temperature. Based on the above, it is obvious that the reactivity between atomic hydrogen and polymer is enhanced and the atomic hydrogen concentration increases with a rise in catalyzer temperature. Therefore, it is assumed that the removal rate rapidly increases with rising catalyzer temperature.

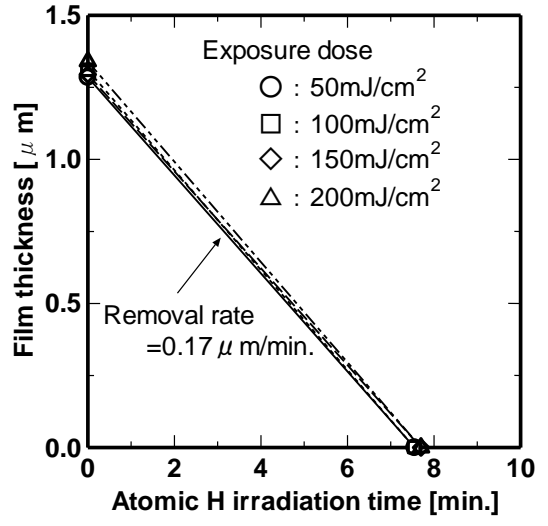

Fig. 7. Relationship between the atomic hydrogen irradiation time and the polymer film thickness with various exposure doses. The catalyzer temperature is $2400^{\circ} \mathrm{C}$.

Figure 5 (a) presents an optical microscope image of the Si substrate surface before polymer removal (before atomic hydrogen irradiation), and Fig. 5 (b) presents that after polymer removal (after atomic hydrogen irradiation). The catalyzer temperature was fixed at $2400^{\circ} \mathrm{C}$, and the exposure dose to polymer was fixed at $100 \mathrm{~mJ} / \mathrm{cm}^{2}$. After atomic hydrogen irradiation, no residual layer of polymer was observed. This result was similar for the entire area of the substrate. Figure 6 presents XPS spectra of the $\mathrm{Si}$ substrate surface before polymer coating, the polymer surface coated onto the Si substrate, and the Si substrate surface after polymer removal. Results indicated that the intensity of the $\mathrm{C}_{1 s}$ peak $(284 \mathrm{eV})$ in the Si substrate after polymer removal is approximately equal to that in the Si substrate before polymer coating. In addition, peaks of $\mathrm{S}_{\mathrm{i} 2 s}(150 \mathrm{eV})$ and $\mathrm{Si}_{2 p}(100 \mathrm{eV})$ were observed at the $\mathrm{Si}$ substrate surface after polymer removal. Thus, it is concluded that atomic hydrogen can remove cured polymer without leaving a residual layer.

Figure 7 depicts the relationship between the atomic hydrogen irradiation time and the polymer film thickness with various exposure doses. The catalyzer temperature was fixed at $2400^{\circ} \mathrm{C}$. Polymers with exposure doses of 50,100, 150, and $200 \mathrm{~mJ} / \mathrm{cm}^{2}$ were removed by atomic hydrogen. The removal rates (= initial film thickness / polymer removal time) were $0.17 \mu \mathrm{m} / \mathrm{min}$ independent of exposure dose. This removal rate is comparable to the removal rate of $0.1 \mu \mathrm{m} / \mathrm{min}$ for generally $i$-line positive-tone novolak resist [10]. It may be that the polymer can be removed faster 
(a)

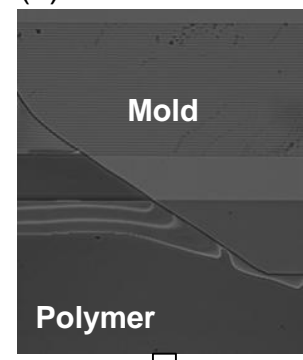

(c)

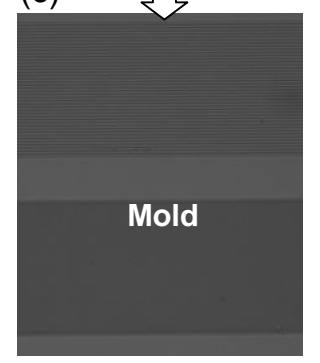

(b)

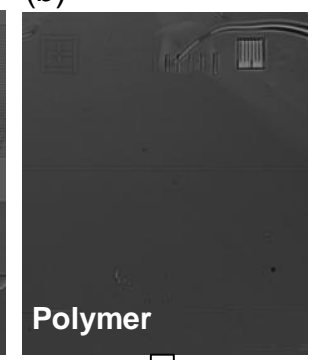

(d)

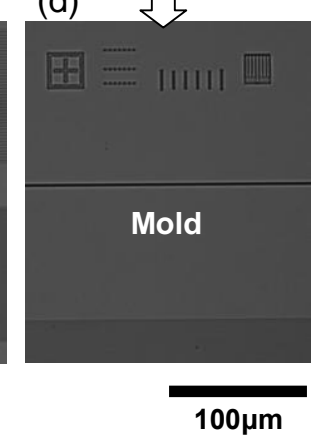

Fig. 8. Optical microscope images $(\times 200)$ of the mold surface before polymer removal ((a), (b)), and after polymer removal ((c), (d)). The catalyzer temperature is $2400^{\circ} \mathrm{C}$. In photograph (a), polymer adheres to part of the mold. In photograph (b), polymer adheres to the entire area of the mold.

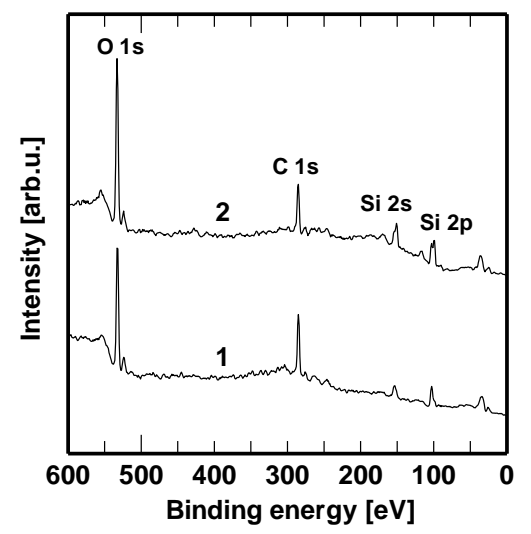

Fig. 9. Wide scan XPS spectra of (1) the mold (with micro-asperity) surface after polymer removal, and (2) the Si substrate surface after polymer removal.

because the maximum removal rate of the novolak resist can reach $2.5 \mu \mathrm{m} / \mathrm{min}$ [7] by optimizing the atomic hydrogen irradiation conditions.

\section{3-2. Removing the Polymer Adhering to the Mold} Using Atomic Hydrogen

We investigated the removal characteristics of the polymer adhering to the mold, and estimated the mold cleaning performance of atomic

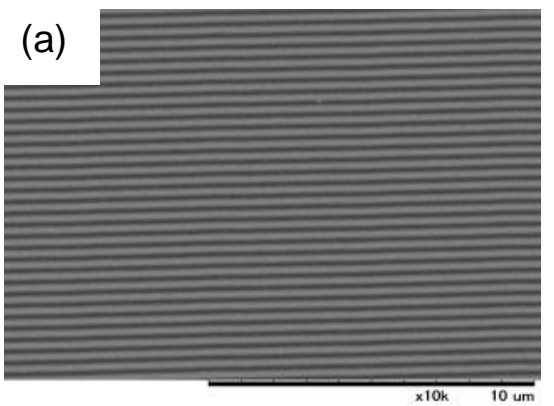

(b)

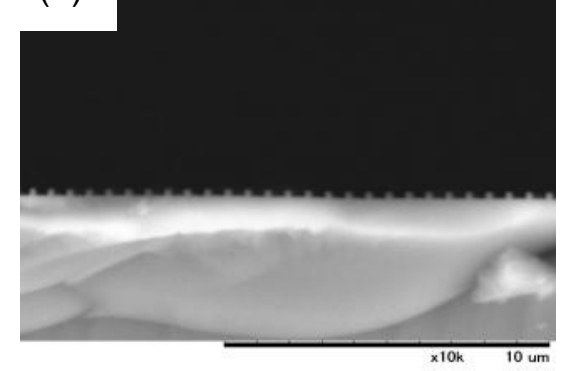

Fig. 10. SEM images $(\times 10000)$ of (a) the mold surface after polymer removal, and (b) the cross sectional surface after polymer removal.

hydrogen. Figures 8 (a) and (b) present optical microscope images of the mold surfaces before the adhering polymer was removed (before atomic hydrogen irradiation), and Figs. 8 (c) and (d) present those after the adhering polymer was removed (before atomic hydrogen irradiation). The catalyzer temperature was fixed at $2400^{\circ} \mathrm{C}$. In Figs. 8 (c) and (d), polymer adhering to a large area of mold was removed by atomic hydrogen. Figure 9 presents the XPS spectra of the mold surface and the $\mathrm{Si}$ substrate surface after polymer removal. Peaks of $\mathrm{Si}_{2 s}$ and $\mathrm{Si}_{2 p}$ were observed in the mold surface after polymer removal. Moreover, peak intensities of $\mathrm{C}_{1 s}, \mathrm{Si}_{2 s}$, and $\mathrm{Si}_{2 p}$ were approximately equal to those in the $\mathrm{Si}$ substrate after polymer removal. Figure 10 (a) and (b) present SEM images of the mold surface (the cross sectional mold surface) after polymer removal. No residual polymer was observed even on the micro-asperity portion of the mold. Based on these results, we conclude that atomic hydrogen can remove adhering polymer from the mold and can clean the mold. In the following section, we explain how the polymer that adhered to the micro-asperity portion of the mold was removed, similar to the polymer coated onto the Si substrate without micro-asperity patterns.

When the velocity of atomic hydrogen follows 
the Maxwell-Boltzmann distribution, the mean free path $\lambda$ of atomic hydrogen is given by equation (1).

$$
\lambda=\frac{1}{\sqrt{2} Q n},
$$

where $Q$ is the collision cross section and is given by equation (2) for collisions of heterogeneous molecules or atoms.

$$
Q=\pi\left(r_{A}+r_{B}\right)^{2}
$$

Here, $r_{\mathrm{A}}$ is the radius of the atom (or molecule), $r_{\mathrm{B}}$ is the radius of the other atom (or molecule), and $n$ is the volume concentration of gas. In the following discussion, we consider the collisions between atomic hydrogen and $\mathrm{N}_{2}$ molecules because $\mathrm{N}_{2}$ occupies $90 \mathrm{vol} . \%$ of the working gas. The radius of atomic hydrogen is $52.9 \mathrm{pm}$, and that of the $\mathrm{N}_{2}$ molecule is $189 \mathrm{pm}$. The $n$ is estimated by total pressure in the chamber $(21.3 \mathrm{~Pa})$ and temperature. The temperature assumed is R.T., and the calculated $\lambda$ is $0.75 \mathrm{~mm}$. This $0.75 \mathrm{~mm}$ is much smaller than the distance between the catalyzer and the substrate (mold) $(100 \mathrm{~mm})$. There are 130 collisions between atomic hydrogen and $\mathrm{N}_{2}$ molecules when the distance between the catalyzer and the substrate (mold) is $100 \mathrm{~mm}$. From this estimate, it is assumed that the atomic hydrogen near the mold surface moves in various directions due to collisions with $\mathrm{N}_{2}$ molecules. The atomic hydrogen penetrated obliquely into the mold and can attack a sidewall of micro-asperity patterns on the mold. Therefore, it is assumed that the polymer adhering to micro-asperity patterns on the mold was removed by atomic hydrogen.

\section{Conclusion}

We investigated the removal of UV curable polymer using UV-NIL by atomic hydrogen, which was generated by the catalytic decomposition of $\mathrm{H}_{2}$ molecules in $\mathrm{H}_{2} / \mathrm{N}_{2}$ mixed gas $\left(\mathrm{H}_{2}: \mathrm{N}_{2}=10: 90 \mathrm{vol} . \%\right)$, using a tungsten hot-wire catalyzer. The cured polymers were removed by atomic hydrogen without leaving a residual layer. The removal rate rapidly increased with rising catalyzer temperature because of enhanced the reactivity between atomic hydrogen and polymer and increased atomic hydrogen concentration due to a rise in catalyzer temperature. When the hydrogen partial pressure was $2.13 \mathrm{~Pa}$, the catalyzer temperature was $2400^{\circ} \mathrm{C}$, the distance between the catalyzer and the substrate was $100 \mathrm{~mm}$, and the initial substrate temperature was R.T., polymers with exposure doses of 50 to
$200 \mathrm{~mJ} / \mathrm{cm}^{2}$ were removed at a rate of $0.17 \mu \mathrm{m} / \mathrm{min}$ regardless of exposure dose. The polymer, which adhered into micro-asperity patterns on the mold, was removed by atomic hydrogen. When the distance between the catalyzer and the substrate was $100 \mathrm{~mm}$, it was assumed that the atomic hydrogen obliquely penetrates into the mold due to approximately 130 collisions with $\mathrm{N}_{2}$ molecules. Therefore, the polymer that adhered to micro-asperity patterns on the mold was effectively removed by atomic hydrogen.

\section{References}

[1] S. Y. Chou, P. K. Krauss, and P. J. Renstrom, Appl. Phys. Lett., 67 (1995) 3114.

[2] Y. Hirai, J. Photopolym. Sci. Technol., 15 (2005) 475.

[3] M. Colburn, S. Johnson, M. Stewart, S. Damle, T. Bailey, B. Choi, M. Wedlake, T. Michaelson, S. V. Sreenivasan, J. Ekerdt, and C. G. Wilson, Proc. of SPIE 3676 (1999) 378.

[4] N. Sakai, J. Photopolym. Sci. Technol., 22 (2009) 133.

[5] Y. Hirai, S. Yoshida, A. Okamoto, Y. Tanaka, M. Endo. S. Irie, H. Nakagawa, and M. Sasago, $J$. Photopolym. Sci. Technol., 14 (2001) 457.

[6] N. Fujii, T. Tanabe, T. Hirasawa, H. Kawata, N. Sakai, and Y. Hirai, J. Photopolym. Sci. Technol., 22 (2009) 181.

[7] M. Yamamoto, H. Horibe, H. Umemoto, K. Takao, E. Kusano, M. Kase, and S. Tagawa, Jpn. J. Appl. Phys., 48(2) (2009) 026503.

[8] M. Yamamoto, T. Maruoka, Y. Goto, A. Kono, H. Horibe, M. Sakamoto, E. Kusano, H. Seki, and S. Tagawa, J. Electrochem. Soc., 157(3) (2010) H361.

[9] H. Horibe, M. Yamamoto, E. Kusano, T. Ichikawa, and S. Tagawa, J. Photopolym. Sci. Technol., 21(2) (2008) 293.

[10] M. Yamamoto, T. Maruoka, A. Kono, H. Horibe, and H. Umemoto, Appl. Phys. Express., 3(2) (2010) 026501.

[11] T. Maruoka, Y. Goto, M. Yamamoto, H. Horibe, E. Kusano, K. Takao and S. Tagawa, J. Photopolym. Sci. Technol., 22(3) (2009) 325

[12] A. Izumi, and H. Matsumura, Jpn. J. Appl. Phys., 41 (2002) 4639.

[13] H. Umemoto, K. Ohara, D. Morita, Y. Nozaki, A. Masuda, and H. Matsumura, J. Appl. Phys., 91 (2002) 1650.

[14] H. Umemoto, 7th Cat-CVD Kenkyukai Koenyokosyu, (2010) 66 [in Japanese]. 